\title{
Publisher Correction: Non-canonical function of IRE1 $\alpha$ determines mitochondria-associated endoplasmic reticulum composition to control calcium transfer and bioenergetics
}

Amado Carreras-Sureda, Fabián Jaña, Hery Urra, Sylvere Durand, David E. Mortenson, Alfredo Sagredo, Galdo Bustos, Younis Hazari, Eva Ramos-Fernández, Maria L. Sassano, Philippe Pihán, Alexander R. van Vliet, Matías González-Quiroz, Angie K. Torres, Cheril Tapia-Rojas, Martijn Kerkhofs, Rubén Vicente, Randal J. Kaufman (D), Nibaldo C. Inestrosa, Christian Gonzalez-Billault, R. Luke Wiseman (D), Patrizia Agostinis, Geert Bultynck, Felipe A. Court (D), Guido Kroemer, J. César Cárdenas and Claudio Hetz (1)

Correction to: Nature Cell Biology, https://doi.org/10.1038/s41556-019-0329-y, published online 20 May 2019.

In the version of the article originally published, co-author R. Luke Wiseman was incorrectly assigned affiliation footnote number 8 in the author list. The correct affiliation number is 7, corresponding to the Department of Molecular Medicine, The Scripps Research Institute, La Jolla, CA, USA. Additionally, the Supplementary Information PDF contained the wrong Supplementary Figures and legends. The Supplementary Information PDF was replaced on 4 June 2019, and the affiliation error was corrected in the PDF and HTML versions of the paper. 